\title{
Self correction of refractive error among young people in rural China: results of cross sectional investigation
}

\author{
(ब) $(\mathbb{N} \Theta$ OPEN ACCESS
}

\author{
Mingzhi Zhang director ${ }^{1}$, Riping Zhang head of refraction service ${ }^{1}$, Mingguang He head of preventive \\ ophthalmology group ${ }^{2}$, Wanling Liang research associate ${ }^{1}$, Xiaofeng Li research associate ${ }^{1}$, \\ Lingbing She research associate ${ }^{1}$, Yunli Yang research associate ${ }^{1}$, Graeme MacKenzie principal \\ scientist $^{34}$, Joshua D Silver director ${ }^{5}$, Leon Ellwein special volunteer ${ }^{6}$, Bruce Moore professor ${ }^{7}$, \\ Nathan Congdon professor ${ }^{2}$
}

${ }^{1}$ Joint Shantou International Eye Centre, Shantou, People's Republic of China; ${ }^{2}$ Zhongshan Ophthalmic Centre, Sun Yat Sen University, State Key Laboratory and Division of Preventive of Ophthalmology, 54 Xianlie S Road, Yuexiu, Guangzhou, China 510060; ${ }^{3} \mathrm{Adlens,} \mathrm{Oxford} \mathrm{OX2} \mathrm{8AH,} \mathrm{UK;}$ ${ }^{4}$ Nuffield Laboratory of Ophthalmology, John Radcliffe Hospital, Oxford OX3 9DU; ${ }^{5}$ Centre for Vision in the Developing World, St Catherine's College, Oxford OX1 3UJ; ${ }^{6}$ National Eye Institute, Bethesda, MD, USA; ${ }^{7}$ New England College of Optometry, Boston, MA

\begin{abstract}
Objective To compare outcomes between adjustable spectacles and conventional methods for refraction in young people.

Design Cross sectional study.

Setting Rural southern China.

Participants 648 young people aged 12-18 (mean 14.9 (SD 0.98)), with uncorrected visual acuity $\leq 6 / 12$ in either eye.

Interventions All participants underwent self refraction without cycloplegia (paralysis of near focusing ability with topical eye drops), automated refraction without cycloplegia, and subjective refraction by an ophthalmologist with cycloplegia.
\end{abstract}

Main outcome measures Uncorrected and corrected vision, improvement of vision (lines on a chart), and refractive error.

Results Among the participants, 59\% (384) were girls, 44\% (288) wore spectacles, and $61 \%$ (393/648) had 2.00 dioptres or more of myopia in the right eye. All completed self refraction. The proportion with visual acuity $\geq 6 / 7.5$ in the better eye was $5.2 \%$ (95\% confidence interval $3.6 \%$ to $6.9 \%)$ for uncorrected vision, $30.2 \%(25.7 \%$ to $34.8 \%)$ for currently worn spectacles, $96.9 \%$ (95.5\% to $98.3 \%$ ) for self refraction, $98.4 \%$ (97.4\% to $99.5 \%)$ for automated refraction, and $99.1 \%$ (98.3\% to $99.9 \%$ ) for subjective refraction $(\mathrm{P}=0.033$ for self refraction $v$ automated refraction, $\mathrm{P}=0.001$ for self refraction $v$ subjective refraction). Improvements over uncorrected vision in the better eye with self refraction and subjective refraction were within one line on the eye chart in $98 \%$ of participants. In logistic regression models, failure to achieve maximum recorded visual acuity of $6 / 7.5$ in right eyes with self refraction was associated with greater absolute value of myopia/hyperopia $(P<0.001)$, greater astigmatism $(P=0.001)$, and not having previously worn spectacles $(P=0.002)$, but not age or sex. Significant inaccuracies in power ( $\geq 1.00$ dioptre) were less common in right eyes with self refraction than with automated refraction $(5 \% \vee 11 \%, P<0.001)$

Conclusions Though visual acuity was slightly worse with self refraction than automated or subjective refraction, acuity was excellent in nearly all these young people with inadequately corrected refractive error at baseline. Inaccurate power was less common with self refraction than automated refraction. Self refraction could decrease the requirement for scarce trained personnel, expensive devices, and cycloplegia in children's vision programmes in rural China.

\section{Introduction}

Much recent attention has focused on the classroom as a locus of activities to promote children's health as well as learning. The World Health Organization's Global School Health Initiative states that schools should "foster health and learning." Among the goals of Unesco's Education for All programme is "Ensuring that the learning needs of all young people ... are met through equitable access to appropriate learning."

Poor vision is a major barrier to achieving a healthy and educationally sustaining school environment for children in many regions of the world. The world's leading ${ }^{3}$ and most easily remedied cause of poor vision among children is refractive error. Spectacles provide a safe and inexpensive solution to this problem. Nonetheless, a WHO supported series of studies among schoolchildren in Africa, Latin America, and Asia has shown that $10 \%$ of children in the developing world require refractive correction. ${ }^{4-12}$ Among secondary schoolchildren in rural China, $60 \%$ require spectacles, ${ }^{12}{ }^{13}$ but two thirds of these are not currently benefiting from appropriate vision correction ${ }^{12} 14$ because of a failure to own or wear spectacles or because they 
wear spectacles that fail to provide adequate vision. ${ }^{15}$ Among Chinese secondary schoolchildren, over $95 \%$ of poor vision ( $\leq 6 / 12$ in either eye) is caused by inadequately corrected refractive error. ${ }^{12}{ }^{13}$ For India, the range is $61-82 \% .{ }^{78}$

Myopia ("near sightedness") is the most common refractive error among school aged children and is strongly associated with self reported poor visual function in this group. ${ }^{13}$ Correction of even modest amounts of myopia improves children's visual function. ${ }^{16}$ Various barriers, however, prevent many children with refractive error from attaining optimum vision and achieving the classroom success envisioned by the WHO and Unesco initiatives. These include the cost of spectacles, ${ }^{17}$ concern about cosmetic appearance,$^{18}$ and an inadequate understanding of the benefits of refractive correction among children, parents, and teachers. ${ }^{14}{ }^{19}$ An important problem in many areas, though, is lack of access to refractive services capable of providing accurate spectacles..$^{15} 17$

Vision screening is effective in identifying children who can benefit from refractive correction. ${ }^{20}{ }^{21}$ Current means of correcting vision in children detected by such programmes, however, are resource intensive. Subjective refraction by a trained professional, the current standard of care, depends on local availability of skilled practitioners. Those few studies that have examined the issue ${ }^{22} 23$ suggest that resource poor areas often fall short of the WHO recommended standard for 2010 of one refractionist per 100000 population. ${ }^{24}$ Automated refraction requires access to expensive machines, which must be adequately maintained and calibrated. Retinoscopy is a less expensive technology potentially suitable for use in the developing world ${ }^{25}$ but the need for rigorous training has limited its use there. Accuracy of automated and retinoscopic refraction in children depends on paralysis of accommodation (the ability to focus on near objects) with cycloplegic drugs, ${ }^{26}$ which also dilate the pupil. Accommodation will otherwise produce a falsely myopic refractive power in children. These preparations must be maintained under sterile conditions and can be associated with unwanted side effects ${ }^{27}$ and refusal of services.

Adjustable devices offer a novel approach to vision correction in areas with few resources. Some, such as the focometer, ${ }^{28} 29$ are capable only of measuring but not correcting refractive error. Adjustable spectacles not only allow the user to adjust the power of each lens independently to achieve optimal vision but can also be worn as a corrective device. This potentially allows refractive correction programmes to occur entirely within the classroom and without the need for specialised professionals. Most published studies on adjustable spectacles have been carried out on adults and have suggested that good vision can be achieved. ${ }^{28} 30$

We compared visual acuity and refractive power obtained by young people using adjustable spectacles (Adspecs, Adaptive Eyecare, Oxford, UK) with two other refraction modalities. Subjective refraction after cycloplegia by a trained vision professional is the standard of care in refraction screening, while automated refraction without cycloplegia is an alternative approach for settings where trained refractionists are unavailable. This initial study was not designed to evaluate the use or acceptance of the spectacles among young people.

\section{Methods}

Between 30 March and 27 May 2010, two to three classes of about 60 pupils each were selected at random from junior high school years 1 and 2 (ages 14-16) at each of six schools in rural Chaoshan, a relatively poor region of eastern Guangdong Province. A total of 27 classes were randomly drawn from a sampling frame of 96 classes. Five schools were located in the township of Xichang (population 9828 in 2008) and one school in Liangying township (population 200000 in 2008). Xichang is located in Dongyuan County, whose per capita gross domestic product ranked 84th out of Guangdong's 104 counties in 2008, while Liangying is located in Chaonan County, which ranked 48th. Both areas are rural, with most of the population working in agriculture. Refractive services are available from a small number of private optical shops and one government eye clinic in each township.

All young people in selected classes who were present at school, had returned parental consent forms, and had unaided visual acuity $\leq 6 / 12$ in one or both eyes were included in the study. We excluded those who could not achieve best corrected visual acuity $\geq 6 / 9.5$ in both eyes with subjective refraction.

The sample size was based on estimating the mean difference in refractive power between two methods of refraction with a $95 \%$ level of significance. With a postulated standard deviation (SD) as high as 1.25 dioptres, detecting a difference of 0.20 dioptres or more with power of $90 \%$ requires a sample size of at least 411, without accounting for paired observations on a single subject. This sample size requirement was greater than that of 263 required to detect a mean difference between refraction methods of 0.02 in the proportion of participants with visual acuity $\geq 6 / 7.5$, with an SD of 0.10 . With an estimated sample of 415 young people, inflated by $30 \%$ to account for potential classroom clustering effects, and assuming $50 \%$ study eligibility on the basis of visual acuity $\leq 6 / 12$, we estimated we needed to include at least 1080 participants.

\section{Visual acuity measurement}

Measurement of distance visual acuity with and without spectacles (if worn) was carried out at $4 \mathrm{~m}$ with a back illuminated Tumbling E logMAR (logarithm of the minimum angle of resolution) chart intended for use at this distance (Precision Vision, La Salle, IL), in an area of each school with luminance of 500-750 lux (Testo 540 light meter, Testo AG, Lenzkirch, Germany). Starting on the top line (equivalent to the $6 / 60$ line), testing proceeded sequentially to the lowest line on which at least four of five letters were correctly identified with first the left and then the right eye covered. Study staff directed participants to maintain a neutral head position and avoid narrowing of the palpebral fissure (squinting to improve vision) in the tested eye. Participants who were unable to read the largest line were tested at $1 \mathrm{~m}$, with the recorded visual acuity divided by four to compensate for the closer distance (for example, reading the 6/60 line at $1 \mathrm{~m}$ would indicate a vision of 6/240).

\section{Examination procedures}

All young people wearing spectacles had the power measured by lensometry (CL100 automatic lensometer, Topcon, Japan). Additionally, all those with uncorrected vision $\leq 6 / 12$ in at least one eye underwent the following examinations in the indicated order:

\section{Self refraction}

The self refraction spectacles contain two lenses filled with a liquid with a refractive index of 1.579 , each consisting of two sealed membranes secured by a frame (fig $1 \Downarrow$ ). The front and rear faces of each deformable lens are protected by rigid plastic covers. The optical power of each lens is determined by the curvature of its surfaces, controlled independently by varying the volume of liquid in the lens with two user controlled pumps, marked with a scale in dioptres (units of refractive power, with 
greater negative value indicating myopia and greater positive value hyperopia). Spherical refractive power ranging from -6.00 to +6.00 dioptres can be obtained by instrument specifications, but no correction of astigmatism is possible. In practice, myopic powers as high as -8.00 dioptres were obtained with some of the adjustable spectacles. The power of each adjustable lens was measured with the lensometer after self refraction.

The head teacher of each class instructed participants and monitored self refraction. Each head teacher received one to two hours of training in the study protocol from study staff, who were generally not vision professionals. Each teacher first performed self refraction on both of his or her own eyes and subsequently monitored self refraction in both eyes of five young people from other classes, who were not study participants and whose data were not recorded. Any mistakes by teachers in explaining or carrying out the protocol were corrected, but no assessment was made of reliability between or within observers or accuracy of self refraction compared with other methods of refraction.

Self refraction was repeated twice for each child, and the results from the second measurement were used in all cases to minimise any learning effect. Vision was measured first in the right eye, with clean spectacles set to zero power and the left eye covered by the participant's hand. A vision chart with identical layout but different order of letters from that used to test unaided acuity was used. Participants then turned the dial on the right side of the adjustable spectacles backwards slowly (creating a minus power lens, for correction of myopia) until letters on the chart were clearest. Vision was measured again. Finally, they turned the dial forward (minimising minus power to prevent eye strain) until the smallest visible line blurred slightly. Visual acuity was measured and, if had not decreased from the previous step, accepted as final. If visual acuity did not improve with self refraction, testing was repeated with the plunger set to +6.00 dioptres (for correction of hyperopia, far less prevalent in this population). The same steps were repeated for the left eye. Self refraction required an average of five minutes for each child.

\section{Autorefraction}

Autorefraction was carried out five times in each eye without cycloplegia, with the mean value recorded. Visual acuity was measured with lenses of the indicated power placed into trial frames (empty frames into which lenses of any desired power can be mounted) with a vision chart with identical layout and different sequence of letters from above. The calibration of the instrument was monitored daily and adjusted as needed.

\section{Cycloplegic subjective refraction}

Cycloplegia was accomplished with two drops of $1 \%$ cyclopentolate administered five minutes apart in each eye. A third drop was given if the pupil still constricted in response to light 15 minutes later. Absence of this response was considered evidence of adequate cycloplegia. After autorefraction was repeated, subjective refraction was performed in each eye separately by a senior refractionist masked to the results of self refraction and non-cycloplegic autorefraction. The starting point was the mean cycloplegic autorefraction and the end point the least myopic power providing best acuity. Visual acuity was measured with a chart with identical layout but different sequence of letters from those used above.

\section{Media and fundus examination}

The media and fundus examination was performed by an ophthalmologist using a direct and indirect ophthalmoscope after pupillary dilatation. Participants with ocular abnormalities were referred for care as needed.

\section{Statistical methods}

Visual acuity in better and worse seeing eyes was measured without correction, with habitual correction (that is, wearing spectacles for participants who owned them), and with correction based on self refraction, non-cycloplegic autorefraction, and cycloplegic subjective refraction. We then calculated the proportion of participants with visual acuity $\geq 6 / 7.5$ in each of the five refraction categories. We used the Wilcoxon matched pairs signed ranks test to assess equality between distributions and proportions of visual acuity, and multiple logistic regression to analyse the association of age, sex, previous use of spectacles, and refractive power (myopia/hyperopia and astigmatism) with failure to achieve visual acuity of 6/7.5 with self refraction.

Refraction power was analysed by using spherical equivalent refractive error (calculated as the spherical power plus half of the cylindrical power, this refers to the average power of a lens across all meridians). We used the Wilcoxon signed ranks test to assess equality between methods, as the normality assumption (tested with the Shapiro-Francia test) was not satisfied.

Differences between cycloplegic subjective refraction, treated as the standard, and the other two methods of refraction were calculated by subtraction and graphically illustrated with Bland-Altman plots.

Analyses were performed with Stata Statistical Software, release 9.0 (StataCorp LP, College Station, TX). Confidence intervals and $\mathrm{P}$ values (significant at $\mathrm{P} \leq 0.05$ ) have been adjusted to account for design effects resulting from the class based sampling plan.

\section{Results}

Among 1613 young people in classes selected for participation and present in school at the time of the study, parental consent was obtained for $1188(74 \%)$ (table $1 \Downarrow$ ). Refusal was more likely among families of boys (adjusted odds ratio 2.04, 95\% confidence interval 1.39 to $2.99 ; \mathrm{P}=0.001$ ), but age did not differ significantly $(1.37 ; \mathrm{P}=0.090)$ between children of families refusing and agreeing to take part.

Among participants with consent, 672 (57\%) were eligible on the basis of having uncorrected visual acuity $\leq 6 / 12$ in one or both eyes (table $1 \Downarrow$ ). Eligible young people were more likely to be girls $(1.85,1.28$ to $2.67 ; \mathrm{P}=0.002)$ but did not differ by age from those ineligible to participate $(1.10 ; \mathrm{P}=0.151)$. Of 672 young people otherwise eligible, we excluded $24(4 \%)$ on the basis of vision uncorrectable to $\geq 6 / 9.5$ with subjective refraction in one or both eyes (amblyopia in 22 participants and congenital cataract in two). The 648 remaining eligible young people, all of whom completed the entire study protocol, form the basis for remaining analyses unless otherwise stated. The study group had a mean age of 14.9 (SD 0.98), 384 (59\%) were girls, and $288(44 \%)$ were wearing spectacles at the time of vision screening.

Figure 2 shows the distribution of spherical equivalent refractive power in right eyes for self refraction and subjective refraction $\Downarrow$. Nearly all those who participated in this study were myopic (negative power), and 61\% (395) had myopia of 2.00 dioptres or more in the right eye on subjective refraction.

Table 2 shows details of visual acuity obtained with the different methods of refraction $\Downarrow$. Without correction, median visual acuity in the better and worse seeing eye was $6 / 24$ and $6 / 30$, respectively. With presenting vision (habitual correction), 
median visual acuity in the better eye was $6 / 12$ and $6 / 15$ in the worse eye, and 6/6 in both better and worse eyes for the three methods of refraction. The five visual acuity distributions all differed from each other $(\mathrm{P}<0.001$, except $\mathrm{P}=0.003$ for the comparison of automated $v$ subjective refraction, Wilcoxon signed ranks test).

The proportion of young people who achieved visual acuity $\geq 6 / 7.5$ in the better seeing eye was $5.2 \%$ (95\% confidence interval $3.6 \%$ to $6.9 \%$ ) in those with uncorrected vision, $30.2 \%$ $(25.7 \%$ to $34.8 \%)$ in those currently wearing spectacles, $96.9 \%$ ( $95.5 \%$ to $98.3 \%$ ) with self refraction, $98.4 \%$ (97.4\% to $99.5 \%$ ) with automated refraction, and $99.1 \%(98.3 \%$ to $99.9 \%)$ with subjective refraction (table 2 ). $\Downarrow$ Within each of these five refraction categories, the proportions who achieved good vision varied considerably across the 27 classes selected for the study. With uncorrected vision, the proportion ranged from none to $18 \%$, with habitual correction from $12 \%$ to $71 \%$, with self refraction from $88 \%$ to $100 \%$, with automated refraction from $93 \%$ to $100 \%$, and with subjective refraction from $94 \%$ to $100 \%$. Although the proportion of the entire sample who achieved good vision in each of the five refractive categories differed from each other $(\mathrm{P} \leq 0.001$, except $\mathrm{P}=0.033$ for self refraction $v$ automated refraction and $\mathrm{P}=0.157$ for automated $v$ subjective refraction, Wilcoxon signed ranks test), the differences between the three methods of refraction were considerably smaller than that between any refraction method and habitual correction.

Uncorrected visual acuity was improved in the better eye by one or more lines on the chart with self refraction in all but 14 (2\%) participants; 13 of these already had visual acuity of $6 / 6$ (and thus could not be further improved.) As shown in table $3 \Downarrow$, improvement over uncorrected visual acuity in the better eye was as great or greater with self refraction than with subjective refraction (the standard) in $545(84 \%)$ participants, while self refraction achieved one line less of vision improvement in 90 (14\%). Only 13 participants (2\%) had improvement of two or more lines greater with subjective refraction than with self refraction.

Among 615 participants with uncorrected visual acuity $\leq 6 / 12$ in the right eye, 23 (4\%) failed to reach visual acuity of 6/7.5 with self refraction. In logistic regression models, predictors of such failure included greater absolute value of spherical power (myopia/hyperopia), higher cylinder power (astigmatism), and not having worn spectacles at presentation. Age and sex were not significantly associated with failure to achieve $6 / 7.5$ vision (table $4 \Downarrow$ ).

Figures 3 shows Bland-Altman plots comparing cycloplegic subjective refractive error against both self refraction and autorefraction in right eyes $\downarrow$. The difference between subjective refraction and autorefraction was significant $(\mathrm{P}<0.001$, Wilcoxon signed ranks test), with a median value of -0.375 dioptres and $95 \%$ of values between -1.875 and 0.00 dioptres. Subjective refraction and self refraction did not differ significantly $(\mathrm{P}=0.256)$, with a median value of 0.00 dioptres and $95 \%$ of values between -0.75 and 0.875 dioptres.

Among right eyes, $5 \%$ had a refractive power differing by $\geq 1.00$ dioptres in either direction (myopic 3\%, hyperopic $2 \%$ ) for self refraction compared with subjective refraction. This result was significantly lower than the corresponding figure of $11 \%$ (all were in the myopic direction) for automated refraction compared with subjective refraction $(\mathrm{P}<0.001$, Wilcoxon signed rank test $)$.

\section{Discussion}

\section{Principal findings of the study}

The first requirement for any refractive technology is the capacity to deliver high quality vision correction to the large majority of people affected. Despite the inability of adjustable spectacles used in our study to correct astigmatism or high levels of myopia and hyperopia, $97 \%$ of rural young people with poor vision could achieve visual acuity $\geq 6 / 7.5$. Though this proportion was significantly less than for subjective refraction and automated refraction without cycloplegia, less than a third of study participants had habitual vision at this level. It has been shown that refractive correction of vision $\leq 6 / 9$ among rural schoolchildren in Mexico led to significant improvement in self reported visual function. No such improvement occurred when participants' baseline vision was $\geq 6 / 7.5$ in the better seeing eye. ${ }^{16}$ Such results suggest that the visual acuity of $6 / 7.5$ achieved by most of our study participants with self refraction is adequate to meet the visual demands of the classroom.

\section{Comparison with other studies}

Our results are generally consistent with one other peer reviewed publication examining self refraction in young people or children. ${ }^{31}$ That study, following the same protocol as the current report and carried out by the same group of investigators, found that $>90 \%$ of young people with poor vision could achieve good visual acuity with self refraction. Participants included in that study, however, were drawn from urban Guangzhou, a relatively wealthy city in China with widespread access to high quality refractive services, and as such were not representative of the populations in which self refraction would likely be used.

\section{Meaning of the study}

Self refraction with adjustable spectacles offers several potential advantages in resource poor areas. Firstly, cycloplegia seems unnecessary to reduce inaccuracies from accommodation (focusing on a nearby target), as is the case with automated refraction. This is probably because of the greater distance between the subject and vision target with self refraction. A mean difference of zero was observed between refractive powers measured with adjustable spectacles and subjective refraction. Automated refraction without cycloplegia, by contrast, resulted in a more negative mean power, indicating inaccuracy in the myopic direction from accommodation. Myopic inaccuracy of $\geq 1.00$ dioptres was present in $16(3 \%)$ of right eyes with self refraction and $70(11 \%)$ with automated refraction. Such inaccuracies can lead to eye strain and poor vision. The ability to refract accurately without cycloplegia can eliminate costs and side effects associated with drug treatments, while potentially increasing uptake of services. Roughly a quarter (26\%) of families in the current study refused permission for their children to undergo cycloplegia. The use of self refraction also obviates the need to purchase and maintain expensive autorefractors. The requirement for highly trained refractionists, who are in short supply in many parts of the developing world, is also reduced. ${ }^{22} 23$

School vision and refractive screening programmes allow children from diverse economic backgrounds to be reached conveniently in areas such as China with high rates of school attendance. Self refraction permits such programmes to be carried out, in principle, by teachers and students or potentially under the supervision of a refractionist with modest training, without need for direct involvement of a highly trained vision 
professional. There are, however, some potential concerns about this model.

Refraction by a vision professional affords the opportunity to carry out a comprehensive ophthalmic examination to detect other causes of poor vision. The importance of such conditions will depend on the setting. Population based studies have shown that refractive error is responsible for most poor vision among schoolchildren in China ${ }^{12}{ }^{13}$ and India. ${ }^{7}$ In our study, 24/672 $(4 \%)$ of children with poor vision in at least one eye had non-refractive causes, but 22 of these were from amblyopia, a condition that responds poorly to treatment in this age range. ${ }^{32}$ Treatable non-refractive causes of poor vision (such as congenital cataract) were detected in only two participants. While these figures might reflect the failure of young people with more severe vision disability to attend traditional schools, this represents the reality of school based vision screening in this rural setting.

Programmes using self refraction could refer children whose vision does not improve optimally for further care. Referrals to existing refractionists and preferential targeting of areas without current access to refractive care could potentially expand delivery of conventional refractive services, allaying the concern that self refraction programmes would undermine local practitioners.

\section{Unanswered question and future research}

Much remains to be learnt about the application of adjustable spectacle technology to young people. Several devices are currently available, and the current results might apply only to the model tested. These devices have a manufacturing cost of about $\$ 19$ (£12, €13), several times the price of the least expensive conventional spectacles. The extent to which this cost might be decreased by redesign and mass production is not known, but there is potential for reduction. Safety of spectacles and stability of refractive power with long term use by young people will be investigated in planned future studies. Given the smaller size of young people compared with adults, the distance between the eyes might also be less, resulting in variable decrease in vision because the pupil does not lie directly in line with the optical centre of each lens. Smaller or adjustable sized frames specifically targeting children might therefore improve vision results. Legal issues regarding the provision of optical devices by lay people such as teachers, which will vary from region to region, remain to be addressed.

\section{Strengths and weaknesses of the study}

Strengths of the current study include the fact that it compared the current standard of care with a novel approach for correcting the world's leading cause of poor vision in children in a highly relevant setting. Teachers supervised and students carried out self refraction in rural schools with a considerable burden of undercorrected myopia. Though $44 \%$ of young people examined already owned spectacles, $70 \%$ of study participants and $39 \%$ of all examined participants had habitual vision $<6 / 7.5$ in the better eye.

The study had several limitations. As noted above, we evaluated the accuracy of self refraction as performed by rural young people but did not assess other practical issues such as cosmetic acceptability, stability of power under conditions of normal wear, or safety of the adjustable spectacles. Because participants were not given the spectacles for long term use, we could not assess the prevalence of eye strain or other subjective complaints. Issues of cosmetic and functional acceptability of adjustable spectacles are of practical importance and are the focus of the next phase of our planned research. A recent randomised trial in China assessed the acceptability to young people of ready-made spectacles,$^{33}$ which might also have modest inaccuracies in power because of the limited range of powers that can practically be kept in stock. The trial found no differences between ready-made and conventional spectacles in any of the measures of vision and participants' satisfaction assessed, though young people at risk for large inaccuracies (about $10 \%$ of potential participants) were ineligible. In our study, only $5 \%$ of participants had self refraction powers differing by $\geq 1.00$ dioptres from subjective refraction. This compares favourably with another study from a nearby area in rural China, in which nearly half of spectacles worn by young people were inaccurate by this amount. ${ }^{15}$

We have found a tendency towards more error in the myopic direction in self refraction compared with subjective refraction among hyperopic participants. ${ }^{31}$ The high prevalence of myopic refractive error in this Chinese cohort might therefore have reduced errors from accommodation.

Teachers would probably carry out vision screening on children themselves if refractive programmes with adjustable spectacles were put into place. Accurate assessment of baseline visual acuity was a key criterion for recruitment into our study and for calculating the success of various methods of refraction in correcting vision. Thus, acuity was measured by research staff in this setting, probably reducing potential inaccuracies. Such inaccuracies would decrease the net effectiveness of self refraction programmes if teachers' sensitivity in identifying children with poor vision was less than $100 \%$. It has recently been reported, however, that, when compared with a vision professional, teachers in rural China have a sensitivity of more than $90 \%$ in identifying children with uncorrected visual acuity $\leq 6 / 12$ in either eye. ${ }^{20}$ This suggests that the reduction in the impact of the programme from inaccurate vision screening by teachers might be modest in this setting.

Despite the study's limitations, our results are promising and provide previously unavailable data on the potential of adjustable spectacles to improve vision in young people in rural schools with a large burden of inadequately corrected refractive error. Further studies are needed to determine if this technology can be implemented in such settings in a manner that is safe, sustainable, and well integrated with existing services.

Contributors: MZZ was responsible for design, logistic support, and data collection. RPZ was responsible for design, supervision, and data collection. MGH and JDS were responsible for conception and design. WLL, XFL, LBS, and YLY were responsible for design and data collection. GM, LE, and BM were responsible for conception and design and analysis of data. NC was responsible for supervision, data collection, data analysis, and writing of the manuscript. All authors critically revised the manuscript and approved the final version and had access to all data. RPZ, GM, LE, and NC are guarantors.

Funding: This study was funded by grant to the Nuffield Laboratory of Ophthalmology (University of Oxford) from the Partnership for Child Development, (Imperial College of Science, Technology and Medicine) under the World Bank's FY2009 Development Grant Facility (DGF)

Window 1. The adjustable spectacles used in this study were provided free of charge by Adaptive Eyecare. The study sponsor played no role in study design and the collection, analysis, and interpretation of data and the writing of the article and the decision to submit it for publication. Competing interests: All authors have completed the ICMJE uniform disclosure form at www.icmje.org/coi_disclosure.pdf (available on request from the corresponding author) and declare: GM has support from Adlens for the submitted work; no financial relationships with any 


\section{What is already known on this topic}

Uncorrected refractive error is the leading cause of poor vision among young people and children in China and the world Self refraction with adjustable spectacles can yield accurate refractive power and good vision in many adults

Because of accommodation (focusing of the eye at near distances), refraction in children is often inaccurate without the use of cycloplegia (paralysis of the ability to accommodate with topical drugs)

\section{What this study adds}

Over $96 \%$ of young people in rural China with poor vision in at least one eye could improve their vision to $\geq 6 / 7.5$ in the better seeing eye by self refraction with cheap adjustable spectacles

Inaccuracy from accommodation during self refraction without cycloplegia was significantly less than for automated refraction with much more expensive devices

organisations that might have an interest in the submitted work in the previous three years, GM is a shareholding employee of Adlens, a company involved in adjustable lenses. JDS is the director of Adaptive Eyecare and the Centre for Vision in the Developing World (a company involved in adjustable lenses) and is a shareholder in Adlens. Ethical approval: The study was approved by the institutional review boards of the Zhongshan Ophthalmic Center (Guangzhou, China), the Joint Shantou International Eye Center (Shantou China), and the University of Oxford. Informed written consent was obtained from at least one parent of each participating child, and the tenets of the Declaration of Helsinki were followed throughout.

Data sharing: No additional data available.

1 World Health Organization. Global School Health Initiative. 2011. www.who.int/school youth_health/gshi/hps/en/index.html.

2 United Nation's Educational, Scientific and Cultural Organization. Education for all program. www.unesco.org/new/en/education/themes/leading-the-international-agenda/educationfor-all/

3 Gilbert $\mathrm{C}$. Changing challenges in the control of blindness in children. Eye 2007;21:1338-43.

4 Maul E, Barroso S, Muñoz SR, Sperduto RD, Ellwein LB. Refractive error study in children results from La Florida, Chile. Am J Ophthalmol 2000;129:445-54.

5 Zhao J, Pan X, Sui R, Muñoz SR, Sperduto RD, Ellwein LB. Refractive error study in children: results from Shunyi District, China. Am J Ophthalmol 2000;129:427-35.

6 Pokharel GP, Negrel AD, Muñoz SR, Ellwein LB. Refractive error study in children: results from Mechi Zone, Nepal. Am J Ophthalmol 2000;129:436-44.

7 Dandona R, Dandona L, Srinivas M, Sahare P, Narsaiah S, Munoz SR, et al. Refractive error in children in a rural population in India. Invest Ophthalmol Vis Sci 2002:43:615-22.

8 Murthy GV, Gupta SK, Ellwein LB, Munoz SR, Pokharel GP, Sanga L, et al. Refractive error in children in an urban population in New Delhi. Invest Ophthalmol Vis Sci 2002;43:623-31.

9 Naidoo KS, Raghunandan A, Mashige KP, Govender P, Holden BA, Pokharel GP, et al. Refractive error and visual impairment in African children in South Africa. Invest Ophthalmol Vis Sci 2003:44:3764-70.

10 He M, Zeng J, Liu Y, Xu J, Pokharel GP, Ellwein LB. Refractive error and visual impairment in urban children in southern China. Invest Ophthalmol Vis Sci 2004;45:793-9.

11 Goh PP, Abqariyah Y, Pokharel GP, Ellwein LB. Refractive error and visual impairment in school-age children in Gombak District, Malaysia. Ophthalmology 2005;112:678-85.

12 He M, Huang W, Zheng Y, Huang L, Ellwein LB. Refractive error and visual impairment in school children in rural southern China. Ophthalmology 2007;114:374-82.

13 Congdon N, Wang Y, Song Y, Choi K, Zhang M, Zhou Z, et al. Visual disability, visual function and myopia among rural Chinese secondary school children: the Xichang Pediatric Refractive Error Study (X-PRES)—report 1. Invest Ophthalmol Vis Sci 2008;49:2888-94.

14 Congdon N, Zheng MW, Sharma A, Choi K, Song Y, Zhang M, et al. Prevalence and determinants of spectacle nonwear among rural Chinese secondary schoolchildren: the Xichang Pediatric Refractive Error Study. Report 3. Arch Ophthalmol 2008;126:1717-23.

15 Zhang MZ, Lv H, Gao Y, Griffiths S, Sharma A, Lam D, et al. Visual morbidity due to inaccurate spectacles among school-children in rural China: the See Well to Learn Well Project, Report \#1. Invest Ophthalmol Vis Sci 2009;50:2011-7.
16 Esteso P, Castanon A, Toledo S, Rito MA, Ervin A, Wojciechowski R, et al. Correction of moderate myopia is associated with improvement in self-reported visual functioning among Mexican school-aged children. Invest Ophthalmol Vis Sci 2007;48:4949-54.

17 Odedra N, Wedner SH, Shigongo ZS, Nyalali K, Gilbert C. Barriers to spectacle use in Tanzanian secondary school students. Ophthalmic Epidemiol 2008;15:410-7.

18 Castanon Holguin AM, Congdon N, Patel N, Ratcliffe A, Esteso P, Toledo-Flores S, et al. Factors associated with spectacle-wear compliance in school-aged Mexican children. Invest Ophthalmol Vis Sci 2006;47:925-8.

$19 \mathrm{Li} \mathrm{L,} \mathrm{Lam} \mathrm{J,} \mathrm{Lu} \mathrm{Y,} \mathrm{Ye} \mathrm{Y,} \mathrm{Lam} \mathrm{DS,} \mathrm{Gao} \mathrm{Y,} \mathrm{et} \mathrm{al.} \mathrm{Attitudes} \mathrm{of} \mathrm{students,} \mathrm{parents,} \mathrm{and} \mathrm{teachers}$ towards glasses use in rural China. Arch Ophthalmol 2010;128:759-65.

20 Sharma A, Li L, Song Y, Choi K, Lam DS, Zhang M, et al. Strategies to improve the accuracy of vision measurement by teachers in rural Chinese secondary schoolchildren: Xichang Pediatric Refractive Error Study (X-PRES) report No 6. Arch Ophthalmol 2008;126:1434-40.

21 Leone JF, Mitchell P, Morgan IG, Kifley A, Rose KA. Use of visual acuity to screen for significant refractive errors in adolescents: is it reliable? Arch Ophthalmol 2010;128:894-9.

22 Bourne RRA, Dineen BP, Huq DMN, Ali SM, Johnson GJ. Correction of refractive error in the adult population of Bangladesh: meeting the unmet need. Invest Ophthalmol Vis Sci 2004;45:410-7.

23 Ramke J, du Toit R, Palagyi A, Williams C, Brian G. Public sector refraction and spectacle dispensing in low-resource countries of the Western Pacific. Clin Experiment Ophthalmol 2008;36:339-47.

24 World Health Organization. Elimination of avoidable visual disability due to refractive errors. WHO, 2000.

25 Donovan L, Brian G, du Toit R. A device to aid the teaching of retinoscopy in low-resource countries [letter]. Br J Ophthalmol 2008;92:294.

26 Fotedar R, Rochtchina E, Morgan I, Wang JJ, Mitchell P, Rose KA. Necessity of cycloplegia for assessing refractive error in 12-year-old children: a population-based study. $\mathrm{Am} \mathrm{J}$ Ophthalmol 2007;144: 307-9.

27 Rengstorff RH, Doughty CB. Mydriatic and cycloplegic drugs: a review of ocular and systemic complications. Am J Optom Physiol Opt 1982;59:162-77.

28 Smith K, Weissberg E, Travison TG. Alternative methods of refraction: a comparison of three techniques. Optom Vis Sci 2010;87:e176-82.

29 Berger IB, Spitzberg LA, Nnadozie J, Bailey N, Feaster J, Kuether C, et al. Testing the FOCOMETER-a new refractometer. Optom Vis Sci 1993;70:332-8.

30 Douali MG, Silver JD. Self-optimised vision correction with adaptive spectacle lenses in developing countries. Ophthalmic Physiol Opt 2004;24:234-41.

31 He M, Congdon N, Mackenzie G, Zeng Y, Silver JD, Ellwein L. The child self-refraction study: results from urban Chinese children in Guangzhou. Ophthalmology 2011;118:1162-9.

32 American Academy of Ophthalmology. Preferred practice patterns: amblyopia. 2011. http //one.aao.org/CE/PracticeGuidelines/PPP_Content.aspx?cid=930d01f2-740b-433e-a973cf68565bd27b.

33 Zeng Y, Keay L, He M, Mai J, Munoz B, Brady C, et al. A randomized, clinical trial evaluating ready-made and custom spectacles delivered via a school-based screening program in China. Ophthalmology 2009;116:1839-45.

Accepted: 17 June 2011

\section{Cite this as: BMJ 2011;343:d4767}

This is an open-access article distributed under the terms of the Creative Commons Attribution Non-commercial License, which permits use, distribution, and reproduction in any medium, provided the original work is properly cited, the use is non commercial and is otherwise in compliance with the license. See: $\mathrm{http}: / /$ creativecommons.org/licenses/by$\mathrm{nc} / 2.0 /$ and http://creativecommons.org/licenses/by-nc/2.0/legalcode. 


\section{Tables}

Table 1 | Distribution of age and sex among young people in rural China in study of refraction. Figures are numbers (percentage) of participants

\begin{tabular}{lcccc} 
& \multicolumn{3}{c}{ Agreed to participate } & \\
\cline { 2 - 4 } $\begin{array}{l}\text { Characteristic } \\
\text { Age (years): }\end{array}$ & Eligible & Ineligiblet & Total & Refused to participate‡ \\
\hline $12-13$ & $31(5)$ & $21(4)$ & $52(4)$ & $12(3)$ \\
\hline 14 & $179(27)$ & $140(27)$ & $319(27)$ & $74(17)$ \\
\hline 15 & $286(43)$ & $239(46)$ & $525(44)$ & $167(39)$ \\
\hline 16 & $130(19)$ & $93(18)$ & $223(19)$ & $137(32)$ \\
\hline $17-19$ & $46(7)$ & $23(5)$ & $69(6)$ & $35(8)$ \\
\hline Mean (SD) & $14.9(0.98)$ & $14.9(0.92)$ & $14.9(0.95)$ & $15.3(0.97)$ \\
\hline Sex: & & & & $279(66)$ \\
\hline Male & $276(41)$ & $288(56)$ & $564(48)$ & $146(34)$ \\
\hline Female & $396(59)$ & $228(44)$ & $624(53)$ & $425(26)$ \\
\hline Total & $672(57)$ & $516(43)$ & $1188(74)$ &
\end{tabular}

*Uncorrected visual acuity $\leq 6 / 12$ in one or both eyes.

†More likely to be girls (adjusted odds ratio $1.85,95 \% \mathrm{Cl} 1.28$ to $2.67 ; \mathrm{P}=0.002)$. Groups did not differ by age $(\mathrm{P}=0.151)$.

$\ddagger$ More likely to be boys $(2.04 ; P=0.001)$ but did not differ significantly by age $(1.37 ; P=0.090)$ compared with those who agreed to take part. 
Table 2| Distribution of Snellen visual acuity (VA) (expressed as number and percentage of children) without correction and with various types of refraction among young people in rural China. Figures are numbers (percentage) of participants

\begin{tabular}{|c|c|c|c|c|c|c|c|c|c|c|}
\hline \multirow{2}{*}{$\begin{array}{l}\text { Visual } \\
\text { acuity } \\
(n=648)\end{array}$} & \multicolumn{2}{|c|}{ Uncorrected VA } & \multicolumn{2}{|c|}{ Presenting VA } & \multicolumn{2}{|c|}{ VA with self refraction* } & \multicolumn{2}{|c|}{$\begin{array}{c}\text { VA with non-cycloplegic } \\
\text { auto-refraction } \dagger\end{array}$} & \multicolumn{2}{|c|}{$\begin{array}{l}\text { VA with cycloplegic } \\
\text { subjective refraction }\end{array}$} \\
\hline & Better eye & Worse eye & Better eye & Worse eye & Better eye & Worse eye & Better eye & Worse eye & Better eye & Worse eye \\
\hline $6 / 6$ & $13(2)$ & - & $78(12)$ & $37(6)$ & $518(80)$ & $441(68)$ & $582(90)$ & $497(77)$ & $602(93)$ & $550(85)$ \\
\hline $6 / 7.5$ & $21(3)$ & - & $118(18)$ & $77(12)$ & $110(17)$ & $169(26)$ & $56(9)$ & $124(19)$ & $40(6)$ & $83(13)$ \\
\hline $6 / 9.5$ & $49(8)$ & - & $104(16)$ & $70(11)$ & $12(2)$ & $19(3)$ & $7(1)$ & $20(3)$ & $6(1)$ & $15(2)$ \\
\hline $6 / 12$ & $59(9)$ & $60(9)$ & $77(12)$ & $97(15)$ & $2(0.3)$ & $9(1)$ & $1(0.2)$ & $3(0.5)$ & - & - \\
\hline $6 / 15$ & $107(17)$ & $79(12)$ & $110(17)$ & $91(14)$ & $2(0.3)$ & $3(0.5)$ & - & - & - & - \\
\hline $6 / 19$ & $69(11)$ & $84(13)$ & $57(9)$ & $85(13)$ & $2(0.1)$ & $4(0.6)$ & - & $2(0.3)$ & - & - \\
\hline $6 / 24$ & $85(13)$ & $88(14)$ & $44(7)$ & $74(11)$ & $1(0.2)$ & $2(0.3)$ & - & - & - & - \\
\hline $6 / 30$ & $68(11)$ & $80(12)$ & $26(4)$ & $45(7)$ & - & - & - & - & - & - \\
\hline $6 / 38$ & $51(8)$ & $65(10)$ & $11(2)$ & $23(4)$ & $1(0.2)$ & - & - & - & - & - \\
\hline $6 / 48$ & $58(9)$ & $86(13)$ & $13(2)$ & $30(5)$ & - & $1(0.2)$ & - & - & - & - \\
\hline $6 / 60$ & $42(7)$ & $71(11)$ & $7(1)$ & $12(2)$ & - & - & $1(0.2)$ & $1(0.2)$ & - & - \\
\hline$<6 / 60$ & $26(4)$ & $35(5)$ & $3(0.5)$ & $7(1)$ & - & - & $1(0.2)$ & $1(0.2)$ & - & - \\
\hline $\begin{array}{l}\text { Median } \\
\text { VA }\end{array}$ & $6 / 24$ & $6 / 30$ & $6 / 12$ & $6 / 15$ & $6 / 6$ & $6 / 6$ & $6 / 6$ & $6 / 6$ & $6 / 6$ & $6 / 6$ \\
\hline Mean VA & 0.325 & 0.241 & 0.554 & 0.449 & 0.951 & 0.919 & 0.975 & 0.943 & 0.984 & 0.965 \\
\hline
\end{tabular}

*Visual acuity in better seeing eye differed between self refraction and both non-cycloplegic automated refraction $(\mathrm{P}<0.001$, Wilcoxon signed ranks test) and cycloplegic subjective refraction $(P<0.001)$.

†Visual acuity in better seeing eye differed between non-cycloplegic automated refraction and cycloplegic subjective refraction $(\mathrm{P}=0.003)$. 
Table 3| Difference in lines of improvement over uncorrected visual acuity comparing self refraction (SR) and cycloplegic subjective refraction (CSR) among young people in rural China. Figures are numbers (percentage) of participants

Difference in lines of improvement Right eye Left eye Better seeing eye

\begin{tabular}{lccc} 
SR $\geq 1$ line better than CSR & $16(3)$ & $15(2)$ & $9(1)$ \\
\hline No difference between SR and CSR & $511(79)$ & $497(77)$ & $536(83)$ \\
\hline SR 1 line poorer than CSR & $108(17)$ & $113(17)$ & $90(14)$ \\
\hline SR 2 lines poorer than CSR & $6(1)$ & $14(2)$ & $7(1)$ \\
\hline SR $\geq 3$ lines poorer than CSR & $7(1)$ & $9(1)$ & $6(1)$ \\
\hline Total & $648(100)$ & $648(100)$ & $648(100)$ \\
\hline
\end{tabular}


Table 4| Logistic regression model of factors potentially associated with failure to achieve visual acuity of 6/7.5 with self refraction in right eyes with uncorrected visual acuity $\leq 6 / 12$ among young people in rural China

\begin{tabular}{lcc} 
Independent variable & Odds ratio (95\% Cl) & P value \\
Spherical power (dioptres) & $2.78(1.84$ to 4.21$)$ & $<0.001$ \\
\hline Cylinder power (dioptres) & $14.1(3.33$ to 59.3$)$ & 0.001 \\
\hline Age (years) & $0.37(0.11$ to 1.20$)$ & 0.093 \\
\hline Female sex & $0.52(0.13$ to 2.10$)$ & 0.348 \\
\hline Not wearing spectacles at baseline & $19.8(3.35$ to 117.2$)$ & 0.002 \\
\hline
\end{tabular}




\section{Figures}

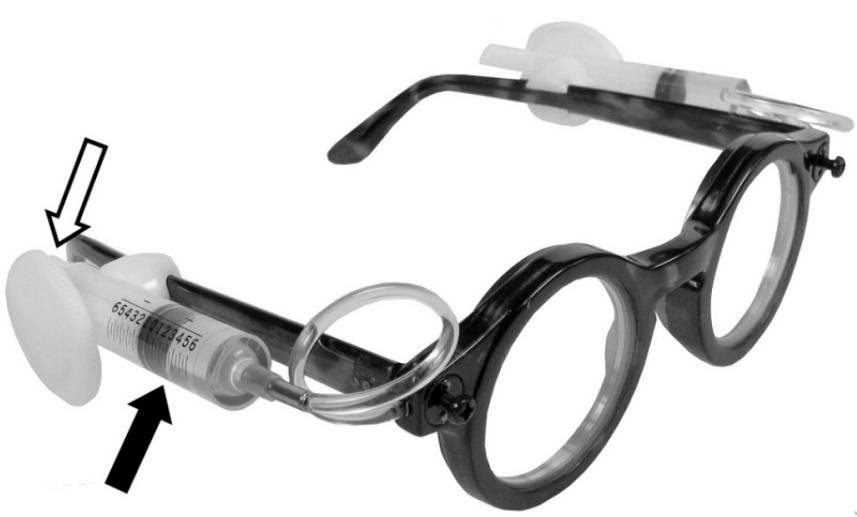

Fig 1 Adjustable spectacles: open arrow indicates adjustment knob, solid arrow indicates dioptre scale on user controlled pump. Lens can be sealed and adjustment mechanism removed after desired power is obtained

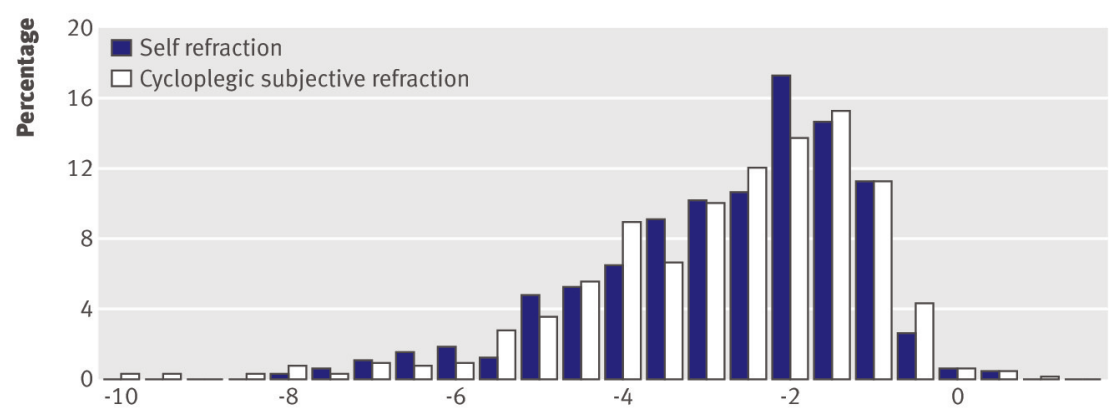

Spherical equivalent refractive error

Fig 2 Distribution of spherical equivalent refractive error in right eye for 648 young people in rural China as assessed by self refraction and cycloplegic subjective refraction. Negative values indicate myopia and positive values hyperopia
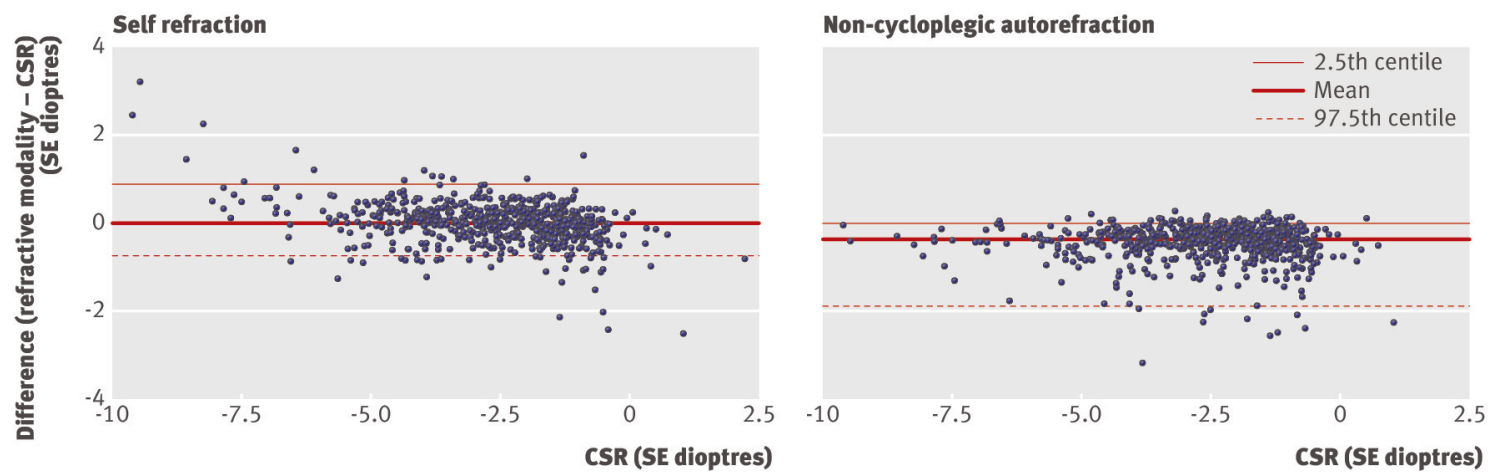

Fig 3 Cycloplegic subjective refractive error (standard care) compared with self refraction and non-cycloplegic autorefraction in the right eye. Horizontal lines represent means with 97.5th and 2.5th centiles (note, two outlying points are not shown on each graph) 